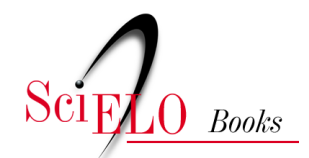

\title{
Comunicación pública en euskera: un proyecto de revitalización
}

\author{
Beatriz Zabalondo Loidi
}

\section{SciELO Books / SciELO Livros / SciELO Libros}

ZABALONDO LOIDI, B. Comunicación pública en euskera: un proyecto de revitalización. In: HABOUD BUMACHAR, M., SÁNCHEZ AVENDAÑO, C., and GARCÉS VELÁSQUEZ, F., eds. Desplazamiento lingüístico y revitalización: reflexiones y metodologías emergentes [online]. Quito: Editorial Abya-Yala, 2020, pp. 41-62. Desafíos en la Diversidad collection, n. 2. ISBN: 978-9978-10-541-2. http://doi.org/10.7476/9789978105726.0003.

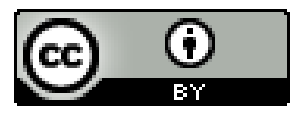

All the contents of this work, except where otherwise noted, is licensed under a Creative Commons Attribution 4.0 International license.

Todo o conteúdo deste trabalho, exceto quando houver ressalva, é publicado sob a licença Creative Commons Atribição 4.0.

Todo el contenido de esta obra, excepto donde se indique lo contrario, está bajo licencia de la licencia Creative Commons Reconocimento 4.0. 


\title{
Comunicación pública en euskera: un proyecto de revitalización ${ }^{1}$
}

Beatriz Zabalondo Loidi²

\begin{abstract}
Resumen
El caso del euskera ha estado presente en muchos congresos y simposios internacionales que han tratado el tema de la revitalización de la lengua desde diferentes puntos de vista. El objetivo de este artículo es mostrar la investigación que se está llevando a cabo en torno a una experiencia de revitalización poco conocida tanto dentro como fuera del País Vasco. Se trata de un proyecto que trata de impulsar el euskera en la comunicación pública de instituciones, empresas y organismos de diversa procedencia, todos ellos conocidos y emblemáticos, dentro del País Vasco meridional. Sin embargo, la mayoría de los medios de comunicación a los que se dirigen estas entidades, y que sin duda son los más poderosos y de mayor audiencia, no trabajan en euskera; además la realidad sociolingüística pocas veces posibilita una comunicación íntegra en euskera, y en muchos casos ni tan siquiera es posible hacerlo en las dos lenguas por igual (castellano y euskera). Este caso de experiencia-investigación está basado en el concepto de Comunidades de Práctica (Wenger, 1998). La investigación estudia cómo se produce el contacto de las lenguas - euskera y castellano - en el ámbito de la comunicación pública, y las limitaciones que se imponen en la práctica diaria. Mediante metodologías cualitativas -2 focus groups a las entidades y entrevistas en profundidad a 7 medios - el grupo de investigación está completando el diagnóstico que contribuirá a buscar espacios para incrementar este tipo comunicación en euskera.
\end{abstract}

1 Agradezco a Soziolinguistika Klusterra la oportunidad de formar parte de este proyecto. Asimismo, agradezco su contribución en este artículo a P. Superbiola, U. Anduaga y A. Basurto (técnicos-investigadores de Soziolinguistika Klusterra); también a E. Apodaka, I. Eskisabel y J. Morales, colegas de la UPV/EHU y compañeros en este viaje sociolingüístico. Alazne Aiestaran y Txiliku me acompañaron en el viaje a Salamanca (ICA, 2018), donde surgió la oportunidad para escribir este artículo. Allí me reencontré con Marleen Haboud y Fernando Ortega, a quienes les debo tanto en mi carrera.

2 Universidad del País Vasco UPV/EHU. 


\section{Introducción}

\section{El euskera, lengua minoritaria}

El euskera es una de las lenguas que se hablan en Euskal Herria (País Vasco); es una lengua minoritaria que habita junto al francés y el español, que son las dos lenguas oficiales y hegemónicas de los estados entre los cuales está dividido este pueblo ${ }^{3}$. Como fruto de las recientes oleadas migratorias, también se hablan otras lenguas dentro de la comunidad vasca — se han registrado ya más de cien-, si bien su uso es mucho más restringido, y exceptuando el inglés, probablemente, son de escasa funcionalidad social. Dichas lenguas también se pueden considerar minoritarias en el ecosistema de Euskal Herria, pero este trabajo se centra en el euskera.

La lengua vasca (euskera) estuvo relegada al ámbito familiar durante siglos, y fue duramente perseguida - a veces abiertamente $\mathrm{y}$ otras de forma no tan evidente- por los gobiernos y autoridades de turno. A pesar de ello, ha conseguido sobreponerse a las mayores adversidades y sobrevivir hasta hoy, con más o menos éxito.

La Real Academia de la Lengua Vasca-Euskaltzaindia, fundada en 1918 a propuesta de Eusko Ikaskuntza-Sociedad de Estudios Vascos, es la Institución Académica oficial que vela por el euskera. Es una institución cuyo cometido es investigar y formular las leyes gramaticales de la lengua vasca, así como el cultivo y protección del euskera.

3 "Euskal Herria, como territorio, está situado en el paralelo $43^{\circ}$, en el extremo occidental de la cordillera de los Pirineos, donde es punto de unión entre la Península Ibérica y el resto de Europa. Lo baña el mar Cantábrico (Golfo de Bizkaia). Ahí es donde el Pueblo Vasco ha permanecido desde tiempo inmemorial como comunidad, con un particular empeño en mantener su propia lengua, el euskera..." (Zallo, 2006, p. 28). 
Según reza en el sitio web de la Academia Vasca:

Euskaltzaindia goza del reconocimiento oficial, con carácter de Real Academia, en España (1976) y, como entidad de utilidad pública, en el ámbito de la República Francesa (1995). Al mismo tiempo, cuenta también con un reconocimiento social generalizado entre la población del país [Euskal Herria]. Todo ello ha propiciado una intensa actividad normativa que se refleja en la estandarización y modernización de la lengua vasca, en especial desde 1968 (Euskaltzaindia).

Desde entonces, hace ya cinco décadas, junto a la normativización del euskera que ha ido proponiendo la Academia, la sociedad civil también ha contribuido enormemente a la modernización de la lengua, sobre todo desde el ámbito educativo — escolar y universitario- y desde los medios de comunicación. En octubre de 2018, la Academia de la Lengua organizó un Congreso Internacional donde académicos de alto prestigio — vascos y extranjeros — celebraron el cincuenta aniversario de la Lengua Vasca Estándar (Euskara Batua).

Si no se hubiera creado el estándar, los vascos no habríamos acordado una gramática, ni reconoceríamos a la Real Academia su autoridad y tampoco se hubiera revitalizado el euskera tanto como se ha revitalizado (a pesar de todas sus defectos y deficiencias). No ha sido fácil ni llano el camino de la estandarización (Anaut, 2013; Arrarats, 2015; Gartzia, 2018; Sarasola, 2016; Zuazo, 2005), pero sin la lengua estándar no se habrían podido crear, escribir ni traducir tantos libros (Torrealdai, 1977 y 2016) ${ }^{4}$ — no habríamos llegado a tener una literatura tan saludable y rica como la actual — ni materiales educativos apropiados para estudiar que, junto a la apuesta rea-

4 Joan Mari Torrealdai, doctor en Sociología y experto en cultura vasca, ha registrado anualmente desde 1975 el número de libros impresos en euskera. Según este autor, en 1976 se publicaron en torno a 95 libros en euskera (1977). En 2016, contabilizó 2061 libros de todo tipo, entre novedades y reimpresiones (2016). 
lizada por un sistema educativo en euskera eficaz han contribuido a que el número de personas que habla y entiende la lengua sea mayor que nunca (VI. Encuesta Sociolingüística, 2016)5. En este sentido, hay que destacar el esfuerzo alfabetizador de la escuela (Sarasola, 2016, p.16; Zallo, 2006, p. 130). Desde luego, los medios de comunicación en euskera serían mucho más débiles, al no disponer de la herramienta apropiada — la lengua que la vehicula_- Estos medios no sólo han permitido informar a los vascos en su propia lengua; sino que además han contribuido a prestigiar la lengua y la cultura, hacerlas más visibles (Zabaleta et al., 2014) .

Aunque antes hemos dicho que el euskera es una lengua minoritaria, en algunos territorios del País Vasco es una lengua oficial junto al español. Sería impensable que el euskera hubiera alcanzado este estatus institucional — parcial— (Zallo, 2006, p.131) sin todo lo que ha conllevado el proceso de estandarización y, por ende, no habría llegado a ser lengua de trabajo y de servicio en la administración y en tantas otras instituciones públicas y privadas (Zuazo, 2005).

A pesar de todos los logros conseguidos, el euskera se encuentra todavía con serias dificultades para hacerse valer en muchos sectores y ámbitos de la vida social vasca, y es por ello que hay todavía muchos proyectos en marcha dedicados a extender y normalizar el uso de la lengua en los espacios menos favorables.

Uno de ellos es el sector organizativo y empresarial, tradicionalmente articulado y vehiculado a través de las lenguas hegemónicas -

5 Desde 1991 hay 200000 vascohablantes más; sobre todo, jóvenes. La mayoría de los escolares estudian en euskera; muchos tienen padres que desconocen la lengua, pero ellos son alfabetizados en el sistema educativo vasco.

6 Además, estos autores han documentado que, en relación a otras lenguas minoritarias de Europa, el euskera es la lengua que más medios de comunicación tiene, después del catalán. 
español y francés; desde hace unos años también el inglés-. Aunque el tejido empresarial vasco ha sido muy activo y rico, hasta hace bien poco la lengua vehicular de trabajo y de comunicación no ha sido el euskera.

Soziolinguistka Klusterra ${ }^{7}$ impulsó el proyecto EraLan para aunar esfuerzos entre entidades organizativas que habían adoptado planes de normalización para el uso del euskera dentro de la empresa y tratar de identificar los factores que inciden en el uso de la lengua en el mundo laboral. Dicho proyecto se materializó en cuatro ediciones - (2006-2008), (2008-2010), (2011-2013) y (2015-2016)—, y fue en la tercera cuando se constituyó expresamente un grupo para estudiar y reflexionar en torno a "Ruedas de prensa y Comunicación pública”. De este grupo surgió la necesidad de investigar y trabajar el tema objeto de estudio que presentamos aquí: Jendaurrean Erabili. Podemos decir que se trata de un caso de revitalización del euskera en un ámbito muy concreto: en la relación profesional que mantienen entidades organizativas y medios de comunicación.

\section{Jendaurrean Erabili}

Jendaurrean Erabili significa "Comunicar/Comunicando en público". Se ha llamado así al proyecto que abarca a organizaciones y empresas — públicas y privadas_implicadas en cuestionar su relación con los medios de comunicación, las cuales difunden sus mensajes, mayormente o solo, en español ${ }^{8}$. Se trata de un proyecto de investigación-acción en torno a la comunicación pública en eus-

7 Significa: Clúster de Sociolingüística Vasco. Véase: http://www.soziolinguistika. eus/es/presentacion.

8 Este proyecto, de momento, se realiza con organismos que trabajan en la parte del estado español. 
kera, liderado por Soziolinguistika Klusterra junto a la Universidad del País Vasco (UPV/EHU).

El proyecto EraLan, arriba mencionado, dejó constancia de que muchas entidades y organizaciones de facto ya tenían cierta experiencia y conocimiento para comunicar (también) en euskera, pues ya lo venían haciendo desde años atrás: algunos como ejercicio de activismo lingüístico y cultural, y las entidades públicas también por una cuestión de derechos y de igualdad lingüística.

El proyecto Jendaurrean Erabili se puso en marcha a finales de 2015. El objetivo específico y principal fue definido de la siguiente manera: Organizar y ofrecer una Comunidad de Práctica que facilite y haga más eficaz la comunicación pública (también) en euskera. El objetivo implícito - que impera en el fondo- es promover y aumentar el uso del euskera en el ámbito público.

La Comunidad de Práctica sería, de este modo, la metodología para crear sinergias entre los agentes participantes, es decir, para compartir y reconocer los problemas que surgen habitualmente en la comunicación pública en euskera, y poder superarlos mediante el aprendizaje mutuo.

\section{Comunidad de Práctica}

Se llaman Comunidades de Práctica (en adelante, CoP) a los grupos de individuos informalmente comprometidos en torno a una experiencia o una pasión compartida (Wenger, 1998; Wenger

9 Por encargo de Soziolinguistika Klusterra, Eduardo Apodaka y Jordi Morales - profesor titular y doctorando respectivamente, del departamento de Psicología Social de la UPV/EHU— diseñaron un proyecto ad hoc cuya finalidad era impulsar y acrecentar el uso del euskera en el ámbito público. Se tuvieron en cuenta estas dos características: a) que los agentes implicados participaran activamente; b) que hubiera interrelación entre la teoría/discurso y la práctica/experiencia. 
et al., 2002; Amin y Roberts, 2008). Los miembros de las CoP se involucran en un proceso de aprendizaje colectivo. Son vehículos eficaces para el intercambio de conocimientos sobre la base práctica de su quehacer, y contribuyen así al conocimiento social. Del mismo modo, estas comunidades contribuyen con su conocimiento a la cohesión y la creación de vínculos identitarios: cuanto mayor sea su presencia y su referencialidad, mayor será su influencia. Es un proceso, por lo tanto, dinámico y vivo, y conviene que la CoP esté activa.

Según Wengler (1998) son tres las características principales que definen a las CoP:

a) Tienen una tarea común — joint enterprise-, en torno a la cual desarrollan su práctica. b) Las personas (en nuestro caso, organizaciones) que forman parte de la $\mathrm{CoP}$ adquieren un compromiso mutuo - mutual engagement - para ayudarse y aprender del conocimiento mutuo. c) Crean y comparten un repertorio o conjunto de recursos de todo tipo — shared repertoire — : un discurso mediante el cual se entienden entre sí, soluciones prácticas, herramientas de trabajo, etc. Pero, sobre todo, crean conceptos y significados sobre esa tarea o práctica común.

En resumen, el objeto de una CoP es compactar la red de individuos, entidades y/o organizaciones existentes en torno a la práctica común que las define (Wengler, 2010). Y el eje de este proyecto - Jendaurrean Erabili - es crear conocimiento (y hábito) para que las entidades públicas y privadas utilicen el euskera en su comunicación pública.

\section{La Comunidad de Práctica de Jendaurrean Erabili}

Según Lave y Wengler (1991), existen varios niveles de participación en las CoP: desde el grupo nuclear de la comunidad a las diferentes capas que la rodean/envuelven, que se van situando en la 
periferia (Figura 1). La parte izquierda de la imagen muestra la idea de dichos autores; la de la derecha es la adaptación para nuestro proyecto. No vamos a detenernos a explicarlas en detalle, pues ya han sido expuestas y explicadas anteriormente en congresos y publicaciones ${ }^{10}$. Baste el esquema para entender que no todas las entidades que constituyen la red de la $\mathrm{CoP}$ participan en igual grado en la actividad, ni con las mismas aspiraciones: cuanto más a la periferia del grupo nuclear se sitúen, su interés y compromiso disminuyen (tal vez alguna entidad solo se acerque a preguntar por el proyecto).

\section{Figura 1}

\section{Esquema de una Comunidad de Práctica}

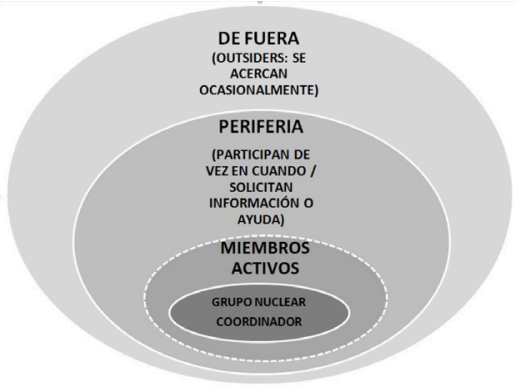

Basado en Lave y Wengler (1991)

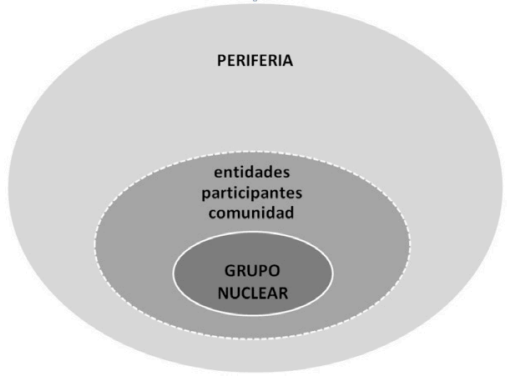

Jendaurrean Erabili (2016)

Realizado por Soziolinguistika Klusterra.

De esta imagen también se deduce que para formar la CoP lo primero que hay que hacer es reunir entidades que formen el Grupo Nuclear.

10 1. BCN-I. Congrés Internacional Revitalització (2017-04-20); 2. Cursos de Verano de la UPV/EHU en Bilbao (Julio, 2018); 3. Soziolingusitika Klusterra (2017). Jendaurrean erabili proiektua. Lehen fasearen txosten laburra, (Resúmen del informe); 4. Apodaka, E; Anduaga, U.; Eskisabel, I. \& Superbiola, P. (2017). "Jendaurrean Erabili Praktika Komunitatea, euskarazko komunikazio publikoari eraginez”, BAT Soziolinguistika aldizkaria, 103 (2), 87-113. 
Impulsar este proyecto fue obra de Soziolinguistika Klusterra: $\mathrm{Al}$ comienzo fueron los profesores Eduardo Apodaka e Idurre Eskisabel de la Universidad del País Vasco (UPV/EHU), junto a Pablo Superbiola y Uxua Anduaga, técnicos de Soziolinguistika Klusterra, quienes estuvieron al frente del proyecto. Es importante destacar que todos ellos eran y son miembros de reconocido prestigio dentro de la comunidad. Este equipo fue el grupo de investigación inicial del proyecto; más tarde se produjeron cambios.

En cuanto a la creación del Grupo Nuclear, se invitó a nueve entidades significativas a que formaran parte del mismo. Fueron elegidas en base a estos dos criterios: que fueran entidades líderes en su propio sector, y que fueran muy activas en la comunicación pública.

\section{Tabla 1}

Entidades integrantes del Grupo Nuclear (noviembre 2015)

\begin{tabular}{|l|l|}
\hline Nombre de la entidad & \multicolumn{1}{c|}{ Actividad } \\
\hline ADEGI & $\begin{array}{l}\text { Asociación de Empresas de Gipuzkoa, cuenta con } \\
\text { más de } 4500 \text { empresas asociadas, y representa a más } \\
\text { de } 75000 \text { personas. }\end{array}$ \\
\hline AEK & $\begin{array}{l}\text { Alfabetatze eta Euskalduntze Koordinakundea (Coor- } \\
\text { dinadora de Euskaldunización y Alfabetización). Aso- } \\
\text { ciación para la promoción y aprendizaje del euskera, } \\
\text { creada en 1976. Se dedica a la enseñanza del euskera } \\
\text {-especialmente a personas adultas-, y a la promo- } \\
\text { ción de su uso, a través de su red de escuelas de euskera } \\
\text { distribuidas por toda Euskal Herria. }\end{array}$ \\
\hline ATHETIC CLUB & $\begin{array}{l}\text { Mayor Club de Futbol de Euskal Herria (más de } \\
\text { B0 000 socios). Fundado en 1898. }\end{array}$ \\
\hline EITB & $\begin{array}{l}\text { Euskal Irrati Telebista (Ente Público Vasco de Radio } \\
\text { y Televisión). }\end{array}$ \\
\hline
\end{tabular}




\begin{tabular}{|l|l|}
\hline ELA & $\begin{array}{l}\text { Sindicato vasco con mayor representación en Euska- } \\
\text { di (no así en Navarra), fundado en 1911. Tiene casi } \\
100000 \text { afiliados. }\end{array}$ \\
\hline $\begin{array}{l}\text { GETXOKO } \\
\text { UDALA }\end{array}$ & $\begin{array}{l}\text { Ayuntamiento del municipio de Getxo, en el territo- } \\
\text { rio histórico de Bizkaia, de casi } 80000 \text { habitantes. }\end{array}$ \\
\hline $\begin{array}{l}\text { GIPUZKOAKO } \\
\text { FORU ALDUNDIA }\end{array}$ & $\begin{array}{l}\text { Diputación Foral de Gipuzkoa, órgano de gobierno del } \\
\text { territorio histórico de Gipuzkoa (Euskal Herria). }\end{array}$ \\
\hline $\begin{array}{l}\text { GUGGENHEIM } \\
\text { BILBAO }\end{array}$ & $\begin{array}{l}\text { Museo Guggenheim de Bilbao. Uno de los mayores mu- } \\
\text { seos de Euskal Herria, y de gran proyección internacional. }\end{array}$ \\
\hline UPV/EHU & $\begin{array}{l}\text { Universidad del País Vasco, es la mayor universidad de } \\
\text { Euskal Herria: 5500 docentes-investigadores y más de } \\
45000 \text { estudiantes. }\end{array}$ \\
\hline
\end{tabular}

Como se puede comprobar, las entidades que formaron este Grupo Nuclear se dedican a actividades muy diferentes entre sí; hay que decir que el grado de compromiso social en su uso del euskera era y es muy diverso, y que todas las organizaciones — sean del sector que sean- actúan voluntariamente en el proyecto.

\section{Desarrollo del proyecto}

Este proyecto se ha diseñado en base a una estructura modular, con objeto de que pudiera ser desarrollado (también) en diferentes fases. Después de unos meses, en el equipo investigador se produjeron algunos cambios: Pablo Superbiola dejó el equipo y Asier Basurto ocupó su lugar, como técnico de Soziolinguistika Klusterra. Por otro lado, se incorporó al grupo Beatriz Zabalondo, profesora de la UPV/EHU.

\section{Primera fase (2016-2017)}

Se planificaron cinco sesiones de una mañana, para el período noviembre 2015-marzo 2016. En la primera sesión, se presenta el pro- 
51

yecto, se avanzan los pormenores y características del mismo, se ubica su procedencia y contexto (su antecedente, el proyecto EraLan), y se constituye el Grupo Nuclear con las entidades arriba mencionadas.

Las siguientes tres sesiones fueron formativas y se articularon según este esquema:

1. Primera parte: Trabajar sobre herramientas conceptuales con profesores de la Universidad del País Vasco. Se impartieron cuatro conferencias:

- "Modelos comunicativos y estrategias comunicativas" (11-XII-2015), con la profesora Beatriz Zabalondo.

- "Prácticas comunicativas y aceptación social" (29-I2016), con el profesor Eduardo Apodaka.

- "Comunicación pública hoy en día: antiguas y nuevas vías" (26-II-2016), con el profesor Patxi Azpillaga. Así como, "Las redes sociales en la comunicación pública: herramienta para analizar el uso del euskera”, impartida por el doctorando Jordi Morales.

2. Segunda parte: Debate e intercambio entre los miembros del grupo, creando espacios para la reflexión y para contar experiencias particulares. Se trabajaron así las relaciones y los referentes comunes.

De esta manera, las sesiones de formación sirvieron para establecer una primera base en comunicación de la CoP. También contribuyeron a tejer relaciones entre los miembros participantes, a reflexionar sobre el sentido de la actividad (comunicar en euskera), poner en valor la actividad y ampliar el conocimiento para la actividad.

Finalizadas estas sesiones formativas, los investigadores realizaron entrevistas en profundidad con cada uno de los agentes del Grupo Nuclear, para elaborar una narración entre el investigador y 
el investigado mediante la técnica de producciones narrativas ${ }^{11}$, con objeto de evaluar la situación (hacer una especie de diagnóstico). Una vez contrastados los relatos con los propios agentes, las narraciones definitivas se utilizaron para formar la base sobre la que se constituyó la CoP.

En junio de 2016 tuvo lugar la última sesión, en la que se daba por concluida la primera fase. En septiembre se presentaron públicamente las conclusiones de la experiencia, y se expresó el deseo de continuar con el proyecto, invitando a su vez a otras entidades a que formaran parte del mismo.

Las ideas principales recogidas en las narraciones, así como en las sesiones formativas se resumen a continuación:

- El euskera tiene un valor distintivo positivo, y las entidades pueden utilizarlo a su favor con diversos objetivos: para fidelizar un público cercano, para ganar legitimidad ante éste, para enriquecer la identidad corporativa de la entidad, etc. De hecho, cada entidad posee públicos específicos ya identificados a los que puede y debe dirigirse en euskera; $y$ haciéndolo también incrementa el uso positivo del euskera. En cualquier caso, el uso público del euskera se entiende como capital. Explicitando esos valores y otros más, se fortalece, extiende y normaliza la comunicación pública en dicha lengua.

- Los grados de legitimidad y normalidad de la lengua (aceptación) varían según el contexto, tipo de entidad, los públicos, etc. En general, se puede afirmar que se da por aceptado y normalizado el uso "inerte" del euskera (en el paisaje, escritos oficiales, notas de prensa...), pero la comunicación activa oral

11 Se trata de una técnica desarrollada por Marcel Balasch y Marisela Montenegro (2003), basada en la epistemología de los conocimientos situados. 
o de mayor interacción presenta mayores dificultades. Aun así, se pueden utilizar diversos recursos para aumentar su legitimidad: calidad del servicio, cumplimiento de la legalidad vigente, etc... Pero, en definitiva, cuantos más agentes comunican en euskera con normalidad, más aceptable resulta.

- Hay entidades que actúan como tractoras en la comunicación pública en euskera (por su referencialidad pública como entidades y porque están implicadas con la comunicación). La labor de estas entidades es necesaria para poder seguir aumentando el uso del euskera en el ámbito público. Muchas de ellas están concienciadas en cumplir con la legalidad, y ejercen el 'bilingüismo sistemático' en todas sus producciones. Este hecho tiene gran repercusión en las zonas menos vascófonas, pues refuerza el grado de aceptabilidad del euskera.

- Sin embargo, no todas las entidades están capacitadas para comunicar en euskera. Una de las mayores dificultades estriba en la falta de preparación y entrenamiento de los propios portavoces y representantes. Por lo tanto, necesitan ayuda y apoyo, para conseguir normalizar la situación. Reunir experiencias concretas en torno a procedimientos para comunicar en euskera puede ayudar en lo siguiente: dar a conocer materiales, grupos y redes de uso, tácticas y trucos, la preparación previa de ruedas de prensa o de otros actos públicos.

- La mayoría de los medios de comunicación tradicionales constituyen un ecosistema propio, donde el euskera apenas tiene cabida. Las entidades los necesitan para dar a conocer su información a sus públicos, pero encuentran dificultades en su actividad comunicativa.

- Es necesaria la colaboración y la mediación entre entidades y medios de comunicación para establecer espacios comunes, consensuar formas... 
- La relación con los medios está cambiando, y en ese cambio hay nuevas oportunidades para el euskera, pero todavía es muy importante la colaboración con los medios tradicionales.

La experiencia se valoró muy positivamente desde Soziolinguistika Klusterra, pues, aunque muy humildemente, se había conseguido poner en marcha la CoP.

\section{Segunda fase (2017-2018)}

Para esta fase el objetivo general se formuló en estos términos: "Promover, facilitar y ayudar eficazmente a las entidades y organizaciones en el uso del euskera en sus comunicaciones públicas, proporcionándoles el material y las vías necesarias para que dicha tarea les resulte más fácil, cómoda y eficaz".

Tras la experiencia con el primer grupo de la CoP — lo que llamamos el Grupo Nuclear- en 2017 se decidió que, para pasar a la siguiente fase, había que afianzar y ampliar la comunidad. El llamamiento para participar en el proyecto tuvo bastante éxito: más de 20 entidades formarían parte de la segunda fase de Jendaurrean Erabili.

El Plan General de trabajo se diseñó en base a cuatro sesiones formativas, con una doble función: a) ofrecer formación e información concreta sobre la comunicación pública en euskera; b) crear espacios para que las entidades de la CoP compartan las estrategias que cada una utiliza para comunicarse con sus públicos a través del euskera.

Al igual que el año anterior, la primera parte de estas sesiones se destinaron a conferencias y/o presentaciones que estuvieran ligadas temáticamente al proyecto: 
- La primera sesión (17-III-2017) trató del liderazgo en la comunicación. Mikel Urdangarin, de Eusliderrak, fue el encargado de la presentación principal. Eusliderrak es un programa para impulsar el uso del euskera en contextos muy diferentes.

- La segunda conferencia (26-V-2017) fue impartida por Luistxo Fernandez (de la empresa Codesyntax), sobre la gestión de las lenguas en las redes sociales, particularmente en Twitter.

- Para la tercera sesión (8-XI-2017) se convocó a Bidane Petralanda, graduada en Medicina, quien presentó su trabajo final de grado: "El rol de la lengua en las relaciones médicopaciente, y los circuitos del euskera en temas de Salud".

- En la cuarta y última sesión (5-XII-2017) participaron dos miembros de la CoP (Xabier Fernández, del Athletic Club de Bilbao; Gorka Quevedo, del sindicato ELA) e Inma Muñoa de la red de ikastolas de Euskal Herria. Disertaron sobre sus modos de gestionar las lenguas en las reuniones multitudinarias de sus respectivas entidades.

Al igual que el año anterior, la segunda parte de las sesiones formativas estuvo también destinada a la discusión y a la puesta en común de dudas, criterios y experiencias entre las organizaciones de la CoP.

Asimismo, se diseñó e implantó una web para ir recogiendo todo el material creado en las sesiones (vídeos, presentaciones, etc.), y ponerlo a disposición de los miembros de la CoP. Dependiendo de sus posibilidades en la participación del proyecto, acceden a más o menos información y material: algunos son miembros de pleno derecho, por su fuerte participación y compromiso activos; otros 
son 'amigos' del proyecto, interesados, pero aún no participan plenamente: son los que se sitúan en la periferia de la CoP (Figura 1).

Además, se abrió una nueva rama de actuación, propuesta e impulsada por la Diputación Foral de Gipuzkoa —integrante del Grupo Nuclear - para trabajar específicamente la posibilidad de aunar un marco de actuación ejemplar en la comunicación pública — en base a la igualdad lingüística — entre entidades de prestigio de la sociedad gipuzkoana. El desarrollo de planes específicos impulsados por alguno de los miembros participantes de la CoP ya había quedado recogido en el proyecto inicial. Este plan específico recibió el nombre de Gipuzkoa Berdinago (Igualdad linguiística en Gipuzkoa $)^{12}$, en el que participaron 18 entidades.

Este plan específico concluyó con una declaración firmada que recoge una serie de hábitos para impulsar el uso público del euskera, siempre con el fin último de buscar un 'mayor equilibrio' en la convivencia lingüística. Hay que notar que se trata de un gran avance, pues el hecho de que tantas entidades representativas de Gipuzkoa convengan en la intención de un mayor uso del euskera en los medios induce a pensar que es un terreno en el que se puede seguir avanzando (también en los demás territorios de Euskal Herria).

Al finalizar 2017, el balance fue muy positivo para Gipuzkoa Berdinago, pero no tanto para Jendaurrean Erabili, pues no se consiguió que la CoP se dinamizara lo suficiente.

Para el siguiente año, 2018, el proyecto se centró sobre todo en la actividad dentro de la CoP, aunque sin descuidar las conferencias

12 Más información: https://www.gipuzkoa.eus/es/-/-gi-puzkoa-berdinagohizkuntza-berdintasuna-elkarlanean 
formativas; además se determinó estudiar más a fondo en torno a la relación que mantienen las entidades y los medios de comunicación.

El objetivo general previsto para activar y dinamizar la CoP se concretó en estos tres objetivos más específicos:

- Fortalecer las relaciones entre las entidades de la CoP, conocerse mejor e intercambiar experiencias entre ellas.

- Promover una participación más activa de las entidades. Crear dinámicas en las que sean receptoras, pero también donantes.

- Propiciar cambios en las prácticas de uso del euskera de las entidades, en su comunicación pública.

En la programación anual del proyecto se idearon dos directrices para contribuir a las relaciones dinámicas y activas de los miembros de la CoP, incentivando el encuentro entre ellos: a) Visitas: se ha propuesto en la CoP que cada entidad invitase a una o dos entidades para que conozcan en vivo y en directo la realidad de la comunicación de la entidad anfitriona. b) También se ha pedido a cada entidad que se proponga unas metas/retos anuales, viables y realizables - siempre relacionados con su uso del euskera en la comunicación pública- Como es de suponer, cada entidad pensó y se 'autoimpuso' metas distintas, en función de su actividad empresarial y sus necesidades.

Continuando con la labor de formación que subyace en el proyecto, también se prepararon cuatro conferencias - testimonios y presentaciones de casos reales del uso del euskera en la comunicación pública - algunas de las cuales habían sido solicitadas por la propia CoP. Fueron las siguientes: 
- “9 preguntas, 9 síndromes, 9 pistas", (23-II-2018), sobre el uso de las lenguas en una experiencia compleja de comunicación pública, fue impartida por Xabier Paya, responsable de Programación Cultural Donostia 2016, Capital Europea de la Cultura.

- "Claves para la activación ciudadana [en el uso del euskera]", (19-IV-2018) con Amaia Balda (de la empresa de Servicios Lingüísticos Emun).

- "Formación de portavoces: dos casos reales de la Diputación Foral de Gipuzkoa" (8-VI-2018), impartida por las profesoras de Ilazki Euskaltegia, centro de enseñanza del euskera (habitualmente a personas mayores).

- "Euskera, marca y publicidad bilingüe" (26-XI-2018), impartida por Estitxu Garai, profesora de Publicidad y Relaciones Públicas de la UPV/EHU.

Además de todas las actividades mencionadas, cabe destacar lo que constituye el estudio que está llevando a cabo el grupo de investigadores en torno a la relación entidades-medios de comunicación. La investigación pretende en primer lugar entender las razonas que subyacen a las relaciones entre estos agentes de la comunicación, sobre todo en cuanto al uso del euskera. Conocer los diferentes puntos de vista ayudará a alcanzar uno de los objetivos más importantes del proyecto Jendaurrean Erabili, que es llegar a negociar y consensuar las condiciones de uso de la lengua minoritaria en la comunicación pública difundida a través de los medios tradicionales. Sin olvidar que el fin último es revitalizar el uso del euskera en el ecosistema mediático, también en el ámbito organizativo y empresarial, y, en última instancia, en la sociedad, en general.

Para tener una visión amplia del contexto en el que las entidades acuden a los medios para su comunicación pública, se plani- 
ficaron dos grupos focales entre los miembros de las entidades de la CoP. Ambos fueron realizados en Donostia el 8 de junio de 2018, en sendos espacios, por las profesoras-investigadoras I. Eskisabel y B. Zabalondo. El objetivo principal era entender con cierta profundidad lo que ocurre en la comunicación con los medios y cuáles son los factores que condicionan el uso de la lengua, siempre desde el punto de vista de las entidades: cómo se sienten las entidades en su relación diaria con los medios, en qué lengua intentan comunicarse, qué obstáculos encuentran, qué materiales proporcionan a los medios, si son suficientes, etc.

Para dar cuenta de los intercambios comunicativos, obviamente había que interpelar también a los medios. El grupo investigador rastreó las audiencias de los medios tradicionales ${ }^{13}$, y en base a los últimos datos, determinó realizar entrevistas en profundidad a los medios o grupos de comunicación de mayor influencia en los territorios de Euskal Herria peninsular. Evidentemente, todos estos medios trabajan y difunden la información mayormente en español; exceptuando uno de ellos, que es bilingüe.

Para las entrevistas, se contó con directores, jefes de redacción o personas de alto nivel representativo de los medios. De los ocho medios de comunicación elegidos para la investigación, solo siete accedieron a la entrevista. Hay que decir que, a pesar de que se les comunicó que las entrevistas se mantendrían bajo estricta confidencialidad - y en los resultados se guardaría el anonimato- no ha resultado una experiencia fácil realizarlas: al principio, algunos medios se mostraron bastante reticentes a conceder las entrevistas, y las citas se fueron demorando hasta bien entrado el año 2019. Aunque

13 Según CIES 2016 — con datos de 2015 - (véase: http://www.ciessl.com/ audienciamedios.htm) y una encuesta propia realizada también en 2015 por el Gobierno Vasco. 
no se han podido conocer los criterios lingüísticos que guían a uno de los medios previstos en el estudio, el grupo de investigadores de Jendaurrean Erabili dio por concluido el tiempo de espera, y cerró este capítulo a mediados de junio de 2019.

\section{Un camino hacia la colaboración}

Nos encontramos a falta de extraer las conclusiones definitivas de estas conversaciones, pero podemos adelantar las siguientes ideas:

- En la relación de las entidades de la CoP con los medios, el conocimiento y la competencia lingüística de los profesionales de ambos lados —comunicadores, portavoces... de las entidades, y periodistas de los medios_ - son factores que afectan directamente para decidir cuál será la lengua de uso en la relación personal, e incluso en la difusión de la información que se realizará a través del medio.

- El periodismo está cambiando, y gracias a Internet y las redes sociales, las entidades se acercan directamente a sus públicos, en la lengua que decidan, cómo y cuándo lo decidan, sin menoscabo de la difusión que realizarán a través de los medios tradicionales. Pero reconocen que aún necesitan a los medios.

- Las entidades no siempre tienen portavoces competentes en euskera, pero intentan buscar otras alternativas para comunicar en euskera: formando a los portavoces, produciendo material bilingüe, aportando intérpretes, etc.

- La relación entidad-medios es más fluida que hace unos años. Las entidades ven que son más aceptadas en el ecosistema mediático comunicando en euskera — sin la exigencia a comunicar en español que provenía de estos medios, en épocas no muy lejanas_- Los medios van aceptando que la sociedad tiene derecho a recibir información en euskera 


\section{1}

- aunque esta demanda varía según el contexto y el territorio-, y en general se puede decir que los medios se percatan de que el euskera está más presente en la sociedad, y lo aceptan así.

- La situación precaria por la que aún están atravesando los medios de comunicación —-debido a la crisis mundial desatada en 2008 - no contribuye a renovar y regenerar las redacciones, ni a formar a los periodistas, por lo que los antiguos profesionales siguen anclados en sus hábitos de trabajo (también lingüísticos). Aun así, se conocen casos de periodistas que han decidido, por sí mismos, formarse en la lengua estándar y están dispuestos y preparados para ejercer el periodismo en la lengua minoritaria.

- Los medios que empiezan a difundir información en euskera son conscientes de que la estandarización de la lengua ha contribuido muy positivamente en la legitimación y la aceptación social, y que ellos deben dar continuación al espacio abierto por los medios que comunican en euskera, buscando a su vez la colaboración de entidades e instituciones.

- En cualquier caso, los cambios que se están produciendo en la sociedad vasca en general inducen a pensar que hay nuevas oportunidades para colaborar y, desde el respeto mutuo, comunicar públicamente en euskera, más y mejor.

- En adelante, hay que seguir trabajando para seguir formando a los profesionales de las entidades y los medios, y procurarles instrumentos lingüísticos y tecnológicos con los que comunicar de manera más ágil y eficaz, para que colaboren en el uso del euskera, siempre teniendo presente que el fin último es revitalizar la lengua (también) desde el ámbito de la comunicación pública. 


\section{Referencias bibliográficas}

VI. Encuesta Sociolingüística (2016). Recuperado de: http://www.euskadi.eus/ contenidos/informacion/argitalpenak/es_6092/adjuntos/Resumen_VI_ Encuesta_Socioling\%C3\%BC\%C3\%ADstica_EAE_\%202016_1.pdf

Amin, A., \& Roberts, J. (2008). Knowing in action: Beyond communities of practice. Research Policy, 37, 353-369. www.sciencedirecte.com.

Anaut, D. (2013). Euskararen kate hautsiak. Hizkuntza zapalkuntzaren memoria. Andoain: Euskal Memoria Fundazioa.

Arrarats, I. (2015). Hutsetik 3.789ra. Jakin 210 (4), 65-78.

Balasch, M., \& Montenegro, M. (2003). Una propuesta metodológica desde la epistemología de los conocimientos situados: Las producciones narrativas. Encuentros en Psicología Social, 1(3), 44-48.

Euskaltzaindia. Real Academia de la Lengua Vasca. Recuperado de: www.euskaltzaindia.eus/es/mas-euskaltzaindia.

Gartzia, P. (2018). San Ignazioren martxa-ren aroa. Zirriborroa euskara batuaren historia intelektualaz. Jakin, 225(2), 45-63.

Gobierno Vasco, Viceconsejería de Política Lingüística (2015). Euskarazko hedabideak. Recuperado de: http://www.hezkuntza.ejgv.euskadi.eus/r43-573/ es/contenidos/ayuda_subvencion/2016_hedabideak_e/es_def/Euskarazko\%20hedabideen\%20kontsumoa\%202015.pdf

Lave, J. y Wengler, E. (1991). Situated learning: legitimate peripherial participation. Cambridge: University Press.

Sarasola, I. (2016). Bitakora kaiera. Donostia: Erein.

Torrealdai, J.M. (1977). Euskal liburugintza 1976an. Jakin, 1, 124-129.

(2018). Euskal liburugintza 2016. Jakin, 226(2), 93-109.

Wenger, E. (1998). Communities of Practice: Learning, Meaning and Identity. Cambridge: Cambridge University Press.

(2010). Communities of Practice and Social Learning Systems: The Career of a Concept. 179-198. En Blackmore, C. (Eds.) Social Learning Systems and Communities of Practice. Springer: London

Wenger, E., McDermott, R., \& Snyder, W. (2002). Cultivating Communities of Practice. Harvard: Business School Press.

Zabaleta, I., Ferre-Pavia, C., Gutiérrez, A., Fernández, I., \& Xamardo, N. (2014). European minority language media and journalism: Framing their marginal reality. International Communication Gazette, 76(3), 275-295.

Zallo, R. (2006). El pueblo vasco. Cultura, historia y sociedad en la era de la diversidad y del conocimiento. Donostia: Alberdania.

Zuazo, K. (2005). Euskara batua. Ezina ekinez egina. Donostia: Elkar. 